\title{
Performing Physics: An Analysis of Design-Based Informal STEAM Education Programs
}

\author{
Simone Hyater-Adams, ${ }^{1}$ Noah Finkelstein, ${ }^{2}$ and Kathleen Hinko ${ }^{3}$ \\ ${ }^{1}$ ATLAS Institute, University of Colorado Boulder, 320 UCB, Boulder, CO, 80309 \\ ${ }^{2}$ Department of Physics, University of Colorado Boulder, 390 UCB, Boulder, CO, 80309 \\ ${ }^{3}$ Department of Physics and Astronomy, Michigan State University, 567 Wilson, East Lansing, MI, 48823
}

\begin{abstract}
Informal educational programs that integrate the arts with sciences technology engineering and mathematics, or STEAM, are growing in prominence, including within informal physics. There are learning and social benefits that can come from blending physics with arts through STEAM education. One benefit that drives this work is the ability of STEAM programs to shift the exclusive culture of typical science learning environments, which is especially relevant to typical physics settings. However, there is room in the literature to understand the different ways we can integrate arts and STEM. This work presents two models for a design-based informal STEAM program that integrates performance art and physics content. We analyze artifacts, video and interview data from a "performing physics" program that was designed and run in the fall of 2015 . We use this analysis to inform a second model of the program that will be run at the end of this summer.
\end{abstract}

\section{INTRODUCTION}

There is a push to address the problem with underrepresentation and equity in STEM careers and classrooms, especially in physics [1]. These efforts usually manifest in two broad ways: building supportive structures within the field or creating new spaces, or counter spaces [2] where underrepresented students can find support. The integration of the arts with STEM, or STEAM, is becoming one method that practitioners and researchers are exploring to create more inclusive STEM learning environments. Many STEAM learning environments are also in the form of informal or outreach programs. These spaces have the potential to become more inclusive of students of color because they 1) may be more engaging than traditional classrooms and 2) can provide opportunities to connect physics to real world applications. In addition, the arts are known to support identity expression and empowerment [3]. The integration of these ideologies and practices can supplement this need within STEM fields, which are known for the value of objectivity and the removal of self.

Physics learning not only encompasses content, but also the ideologies and practices of the field that lead to an identity as a physicist $[4,5]$. Our ongoing work examines what it means for Black folks to identify as physicists, and how specifically, performance art might support this identity. Here we postulate that a STEAM program that sits at the intersection of performance art and physics could provide a physics learning environment that supports the possibility of Black youth building physics identities. In this paper, we outline our first attempt at creating such a program, and the design-based analysis we have used to iterate on the program model. We are seeking to understand the critical elements for a STEAM program that integrates performance arts with physics. We use a design based research approach and report on the elements we learned that were important from our first iteration. We then present a second iteration of the Performing Physics model, as well as postulate a broader model for the design of a program that integrates physics and the performing arts.

\section{PERFORMING PHYSICS PROGRAM DESIGN}

We started developing the design of Performing Physics with the premise that youth would be able to engage positively with the performing arts, as it would allow them to creatively express physics content through their own lens. The first program had a goal of teaching students E\&M content using a project-based activity that culminated into a performance. The program was part of an already existing informal physics program called Partnerships for Informal Science Education in the Community (PISEC). PISEC creates an environment where children and adults can be engaged together in authentic physics practice, which includes exploration, creativity, collaboration, and practices in line with the nature of science, such as prediction, measurement, model making, and evidence-based reasoning [6]. PISEC already has a goal to cultivate physics identities amongst the youth in the program, which makes the space an ideal starting model for the Performing Physics curriculum to build from. In this section, we describe the design principles we developed to modify the PISEC program in order to incorporate performing arts.

\section{A. Design Principles for Performing Physics}

The Performing Physics program aims to teach physics content while encouraging student agency. We chose to integrate performance art because there are many studies linking performance and identity. Performance can also teach skills particularly useful for students of color in physics. Richard Bauman [7] describes performance as involving a consciousness of doubleness where the performer is constantly in negotiation between their own cultural models and the ones around them. This idea mirrors the phenomenon of code switching, where students of color must perform a different identity when in scientific settings. We believe that the integration of performance art with the sciences, and in our case physics, has the potential to help mitigate some of these identity struggles that students of color face when pursuing the field [8]. 
With this in mind, we developed 5 design principles for the Performing Physics curriculum:

1. Embodied practices [9] have been used as an effective means of learning STEM content [10]. These activities provide a more engaging method for understanding physics concepts, which can oftentimes seem abstract and not connected to the real world [11]. Most forms of performance are embodied practices at their root, and therefore, it makes sense to use performance as a tool to create embodied activities in the Performing Physics program [12]. 2. Action of reflection has been identified as a useful practice in physics learning spaces [13]. Having students reflect on the content they are learning can be a metacognitive practice that allows them to identify areas of the content they do or do not fully understand. Reflection is also an important part of many types of performance. In the dance literature, Lerman's Critical Response Process [14] provides a model of structured reflection for group and one on one feedback throughout the creative process. Therefore, the Performing Physics program has activities specifically for reflecting on the day's activities. 3 . Engagement in project based learning has become popular in STEM programming and specifically in physics education. These types of activities are particularly useful for STEAM and informal programs and they allow students to work toward a real world, tangible goal [15]. The performing physics program uses project based learning to work toward the goal of 4. synthesizing content into a culminating performance. Activities that encourage students to synthesize information can be useful assessments of the program's learning goals [16]. 5. Privileging youth agency is important in this informal environment because agency can lead to identity development [17]. We give students autonomy over their projects in Performing Physics and value their personalized methods for completing the project. In performance art, generative knowledge is valued, which validates the knowledge stemming from one's personal experience. In performing physics, we use the generative practice of creating a performance in order to give students agency over their work in the program.

\section{B. Curriculum}

In order to enact these design principles in practice, we developed programming centered around concrete learning goals. Specific activities were also created from the design principles and organized around the learning goals. The overall program and the specific activities were also influenced by the structural and logistical constraints around the existing PISEC partnership model. The program was structured in 3 phases that accomplished three goals: 1) to learn about basic E\&M concepts specific to circuits, 2) to build a set of paddles that create an electric field that can move objects, and 3) to create a performance about the concepts they learned in the first two phases using the paddles.

Phase 1 of the curriculum consisted of typical PISEC activities, which are inquiry based and exploratory, as well as one kinesthetic activity about circuits (design principle 1). Students formed groups to do an activity for the day with their University volunteer, whom we called a University Educator (UE). Each activity included questions that asked students to reflect on the process they used for the activity and the phenomena they were learning about (design principle 2). The students worked with university volunteers who were trained to participate with students in a way that allows them to drive the activities (design principle 5). Phase 2 of the curriculum focused on building electrostatic paddles (design principle 3 and 4). In this phase, students engaged in a project based curriculum where the activities focused on building the paddles. In groups, students built electrostatic paddles through similarly framed activities as those in the inquiry based phase. With the help of UEs, students built an amplifier using capacitors and connected it to paddles. The activities still included questions about content and process. Phase 3 of the curriculum focused on the creation of a performance (design principle 3-5). Students were given storyboards for use in creating a performance using the paddles they built. This activity used a similar framing as the culminating activity in traditional PISEC curriculum, where students are asked to pick a concept or idea that they learned, and express it through a stop action motion video. In this activity, the prompt allowed for any form of performance that could be recorded.

\section{REFLECTIONS}

The first iteration of Performing Physics was successful in providing us areas for feedback and reflection on what it means to integrate the arts with physics. We use an ethnographic approach in our design-based inquiry into the program's model. The program's structure included activities where students created artifacts including notebooks, reflection videos, site videos, feedback surveys, and the final performance video. We draw from these artifacts, in addition to the curriculum planning materials and an interview with the teacher at our partnering school in order to pull out the main areas of reflection that we outline in this section. In order to find these areas, the leading team for the site which consisted of the first and last author, conducted an analysis of the data produced from the program (the artifacts, interview and planning materials) in regular meetings. Many of the artifacts from students were incomplete, due to a struggle we had with attendance at the site, so most of the analysis is based on the other data sources. Through this process, we were able to compare the intent and implementation of the program, and found two critical features of this iteration of the model: 1) the process of developing and maintaining relationships with the partnering community/school 2) training and teaching the creative process.

Teaching Performance: In Performing Physics iteration 1, the curriculum focused more on learning physics content than performance content. This is demonstrated throughout the curriculum in the learning goals for the daily activities, the re- 
flection questions, as well as in the videos students produced. For example, there was only one embodied activity out of the five activities in Phase 1 of the program, and three arts-related reflection questions out of 35 total. The performance phase of the curriculum was designed to be loosely structured so that students had the freedom to create any type of performance they wanted (we did not restrict to a specific art form like dance, skits, or music). With this creative freedom in combination with the paddles they built in the project-based phase, the students were instructed to use a storyboard as a tool to tell a story about something they learned during the program. In practice, these prompts did not provide students with enough support in the process of creating their performance, and students struggled to exercise the agency we intended the activity to provide. We learned that the creative process was not "easy" or "intuitive" to the students in the program, even though they attended a school that emphasized the arts.

For the next iteration, there needs to be more structure around how to create performances, and more scaffolding with prompts and activities aimed at teaching how to create a specific type of performance. We have an idea that transitioning from exclusively physics-based to exclusively performance-based content contributed to what did not go smoothly and now postulate that infusing physics and performance content and practices throughout the program from the beginning would improve the model. Relatedly, our analysis showed that the UEs for Performing Physics iteration 1 were exclusively students from the university with STEM backgrounds. From informal feedback and feedback surveys, it was clear that UEs felt anxious about navigating dance content but felt confident in the physics. Because of the struggle with attendance, UEs also felt awkward about not having that many students to work with. The training for the program required to review all of the activities, but it did not require them to create their own performance. Including this aspect in training is an important change that should be made for iteration 2. Recruiting UEs with a performance arts background can address this concern.

Context: Our analysis also suggests that the context of the first iteration was not as aligned with the program as it needed to be. Our partnering school for iteration 1 was a high school that emphasizes the integration of the arts and science. We took the steps to connect with the school by identifying teachers who would be willing to work with us to develop a partnership. While the teachers we ended up partnering with were excited to work with us, their busy schedules impacted the amount of time we were able to co-plan the program. In the meetings that did occur with the partnering teachers, we talked heavily about the curriculum and physics content, and less about the nuances of the community and the students. Because of this, the program model was not informed by collective goals between us and the partnering teachers, nor some important information about the norms of the community. This communication mismatch led to a miscommunication about the program to students, which was pitched as a "Science Club" instead of an integrated performance and science program. We were able to gather some of these lost details during an informal interview that was conducted with one of the partnering teachers at the end of the program. We learned that providing students with snacks could have been an important feature because the school was located in a food desert. The school also put on regular arts showcases that we could have fit our program into with more planning. We also learned that many students have to work after school and most students only stayed after school for sports, which could have contributed to the decreasing attendance throughout the duration of the program. For iteration 2, we must spend more time learning about the interests, norms, and needs of the students and other stakeholders we look to partner with.

\section{CONCEPTUALIZING THE SECOND ITERATION}

The landscape of STEAM programs sits heavily in the informal and outreach arena with little research to evaluate what the spaces do for students. However, when we narrow to programs that integrate physics content and the performance arts, we find fewer examples in the informal sphere to pull from. From a review of the literature and a search for programs that aren't being studied, we see the landscape of physics and performance learning lies along a spectrum from programs that use physics to serve performances (e.g. wearables in costume design) and programs that use the performance arts to serve physics learning and communication (e.g. kinesthetic activities). These programs include in-school and out-of-school spaces, yet we find no research-based models for designing these spaces to support student identity.

To conceptualize the changes, we plan to make for iteration 2 , we operationalize the STEAM landscape continuum. The goal for our first iteration was to teach physics content to high school students, while using a cumulative performance activity to integrate creativity, agency, and support identity. From our reflections, we learned that shifting from physics to performance content and practices was not the best method for integrating performance into the program. Some of this was due to logistical complications within the context, but also was connected to the curriculum design. In iteration 1, much of the content and practices students engaged with in the program were heavily physics related. For iteration 2, we shift our goal a significant amount towards teaching both physics and performance content and practices in an effort to counteract the misunderstandings students and volunteers had about the first iteration of the program.

Figure 1 shows a schema of how we depict the shifts in the content and practices of Performing Physics for iteration 2 after the analysis of iteration 1 . We plot iteration 1 , in the orange box, very close to the "physics" side of the spectra as a result of our analysis. The learning goals of the program were to teach the physics content practices and content in this iteration focused heavily on the physics content. We aim to have iteration 2 sit in the middle of the spectra, where physics and performance practices and content are blended evenly. 


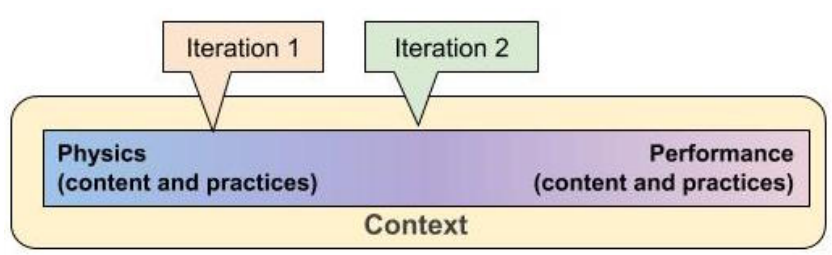

FIG. 1. Our visualization of the spectrum of physics and performance content and practices that can be incorporated into a STEAM program. We plot where we think Performing Physics iteration 1 fell in practice as well as our vision for iteration 2 .

Both spectra sit within the larger context of the partnering community, as our analysis points to context as being one of the most crucial aspects of designing a future program. We see this schema as the beginnings of a model for designing STEAM programs.

\section{PERFORMING PHYSICS ITERATION 2}

Iteration 2 will be a summer camp in collaboration with an education non-profit. It is located in a small and close knit community that has a growing outreach component. The design principles for the program remain the same as iteration 1 ; however, the activities, curriculum and structure of the program are shifting in many ways in order to cultivate more of a performance culture into the physics centered model of iteration 1 . The model for iteration 2 will address the two areas of reflection from iteration 1 through changes in several program features. We have been fostering the relationship with our partnering community for over 6 months, through visits and planning meetings with the organization. Half of the time in the program will be spent on PISEC-like activities, and the other half will be activities that scaffold the process of creating a performance. In addition, the final performance will be framed as the project-based activity in the program. Half of the volunteers recruited have backgrounds in one or more performance art practice. The program will also provide food, be shorter in duration, and target younger students.

The curriculum for the Performing Physics Program is being developed in collaboration with current and past coordinators of PISEC, as well as members of the partnering nonprofit and community. It will build off of the iteration 1 curriculum, but will incorporate more performance content and practices throughout. Students will start the day similar to a dance class, on the floor in a circle, and with a warm up. They will then move to the activities (Phase 1) that will focus on sound, motion, and force concepts in order to integrate more movement-based activities into the physics lessons. For example, students will map dance moves using a motion sensor. They will then shift to a combination of Phases 2 and 3 , working on a performance, which will be the sole projectbased activity. Each day will offer scaffolding with a mini lesson in creating performances, which will shift each day between dances, skits, and music, and end with time to work on the end of program performance. Students will have access to volunteers who identify as performance artists and physicists for the entire time.

In this paper, we contribute to the growing literature on STEAM spaces by reporting on our design based research of the Performing Physics program. We present the design for our first attempt at this, as well as our process and findings from evaluating it. Our future work will include evaluating iteration 2, and further developing the model for physics and performance programs that can serve as a starting point for future researchers and practitioners interested in designing these learning environments. Because this unique intersection of STEAM can potentially recruit and support students of color in physics, we plan to continue this research to understand more about the environment.

We acknowledge NSF GRF, NSF \#1423496 and the NSF JILA Physics Frontier Center for funding this work.
[1] Czujko, R. et.al. Untapped Talent. AIP Report R-444. Stat. Res. Cent. AIP, 2008.

[2] M. Ong, et.al. Counterspaces for women of color in STEM higher education: J. of Res. in Sci. Teach., 2017.

[3] A. Albright, Choreographing difference. Wes. U. Press. 1997.

[4] Z. Hazari and G. Sonnert,. J. of Res. in Sci. Teach., 2009.

[5] W. Chang. Canadian J. of Sci., Math.\& Tech. Ed., 5(1), 2005.

[6] K. Hinko, P. Madigan, E. Miller, N. Finkelstein. PhysRev PER, 12(1), 2016.

[7] R. Bauman, E. Barrow (ed.). Interntl. Ency. of Comm., New York: Oxford U. Press, 1989.

[8] T. Williams et.al. Intersection of Identity and Performing Arts for Black Physicists. PERC 2018. Under Review.

[9] S. Weisberg \& N.Newcombe. Embodied cognition and STEM learning. Cog. Res., 2(1), 38. 2017.

[10] C. Kontra et.al. Physical physics. Cogn. Proc. Vol. 13, D69121, 2012.
[11] Abrahamson, D., \& Lindgren, R. (2014). Embodiment and embodied design. The Cambridge handbook of the learning sciences, 2, 358-376.

[12] Levin, D. (1975). The Embodiment of Performance. Salmagundi,(31/32), 120-142.

[13] A. Miyake et.al. Reducing the Gender Achievement Gap in College Science. Sci., 330(6008), 2010.

[14] L. Lerman \& J. Borstel. Lerman's Critical Response Process. Cont. Quart.. Wint/Sp,2008.

[15] J. Martinez. The Search for Method in STEAM Education. Cham: Springer Int. Pub., 2017.

[16] K. Lundstrom et.al. Teaching and learning information synthesis. Comm. in Info. Lit., 9(1), 4. 2015.

[17] J. Doscher et.al. Sustainability Topics in Physics Education, Science Agency Beliefs and Physics Identity. AAPT, 2015. 